\title{
ORTHOGONAL POLYNOMIALS ON SEVERAL INTERVALS VIA A POLYNOMIAL MAPPING
}

\author{
J. S. GERONIMO AND W. VAN ASSCHE
}

\begin{abstract}
Starting from a sequence $\left\{p_{n}\left(x ; \mu_{0}\right)\right\}$ of orthogonal polynomials with an orthogonality measure $\mu_{0}$ supported on $E_{0} \subset[-1,1]$, we construct a new sequence $\left\{p_{n}(x ; \mu)\right\}$ of orthogonal polynomials on $E=T^{-1}\left(E_{0}\right)(T$ is a polynomial of degree $N$ ) with an orthogonality measure $\mu$ that is related to $\mu_{0}$. If $E_{0}=[-1,1]$, then $E=T^{-1}([-1,1])$ will in general consist of $N$ intervals. We give explicit formulas relating $\left\{p_{n}(x ; \mu)\right\}$ and $\left\{p_{n}\left(x ; \mu_{0}\right)\right\}$ and show how the recurrence coefficients in the three-term recurrence formulas for these orthogonal polynomials are related. If one chooses $T$ to be a Chebyshev polynomial of the first kind, then one gets sieved orthogonal polynomials.
\end{abstract}

I. Introduction. Suppose a sequence of orthogonal polynomials is given with an orthogonality measure supported on a set $E_{0} \subset[-1,1]$. In this paper we will analyze how properties of these orthogonal polynomials, their measure, and the set $E_{0}$ are transformed under a polynomial transformation. In particular we will restrict our class of transformations so that the inverse of $E_{0}$ under the transformation will be a real set. Although special cases of this problem had been considered by Barrucand and Dickinson [5], the general problem was posed and considered in an important paper by Bessis and Moussa [8]. In their paper many of the algebraic properties of the transformed polynomials and their orthogonality measure were discussed. Here we extend their work and apply it to a number of interesting special cases.

If $E_{0}$ is an interval then the inverse image of $E_{0}$ (which is assumed to be real) will in general be a finite number of disjoint intervals, and we are led to the problem of polynomials orthogonal with respect to a measure supported on several intervals. Besides being of interest in its own right (Aptekarev [3], Geronimo and Van Assche [12], Geronimus [13-14], this problem is of interest in numerical analysis when one tries to solve large indefinite linear systems using Richardson iteration (de Boor and Rice [11], Lebedev [18], Saad [23]). Applications of these types of polynomials have also appeared in quantum chemistry (Wheeler [27]) and physics (Pettifor and Weaire [22]). Orthogonal polynomials on several intervals have also appeared in the study of sieved orthogonal polynomials (Al-Salam, Allaway, and Askey [2], Charris and Ismail [9], Ismail [15-17]). Many results involving sieved orthogonal polynomials follow by taking a particular polynomial transformation.

Received by the editors January $5,1987$.

1980 Mathematics Subject Classification (1985 Revision). Primary 42C05.

Key words and phrases. Orthogonal polynomials, recurrence coefficients, Jacobi matrices.

The second author is senior research assistant of the Belgian National Fund for Scientific Research. Permanent address. Katholieke Universiteit Leuven, Departement Wiskunde, Celestijnenlaan 200B, B-3030 Leuven (Belgium) 
In $\S I I$ we give an outline of the general procedure of mapping $E_{0}$ to $E=T^{-1}\left(E_{0}\right)$, where $T$ is a polynomial of degree $N$, and describe how a measure $\mu_{0}$ on $E_{0}$ is transformed to a measure $\mu$ on $E$. We also describe how the associated polynomials and their orthogonality measure transform. In §III we give a relationship between the Jacobi matrices for the orthogonal polynomials on $E_{0}$ and on $E$. §IV lists some properties of the new orthogonal polynomial system. In particular, we give explicit formulas for some of the recurrence coefficients in the new system in terms of those of the old system. We also discuss convergence rates of the coefficients in the recurrence formula and growth rates of the polynomials on the spectrum. In $\S \mathrm{V}$ we describe some of the potential-theoretic properties of the set $E$ and discuss some of the properties of the polynomials associated with the equilibrium measure. In this section we also discuss the Bernstein-Szegö polynomials (Akhiezer [1]). Finally, in $\S \mathrm{VI}$ we discuss sieved orthogonal polynomials.

II. The general procedure. We start off with a probability measure $\mu_{0}$ on a set $E_{0} \subset[-1,1]$. If $\mu_{0}$ has an infinite support, then there exists a unique sequence of orthogonal polynomials $\left\{p_{n}\left(x ; \mu_{0}\right): n=0,1,2, \ldots\right\}$ for which

$$
\int_{E_{0}} p_{n}\left(x ; \mu_{0}\right) p_{m}\left(x ; \mu_{0}\right) d \mu_{0}(x)=\delta_{m, n}, \quad m, n \geq 0 .
$$

We map the interval $[-1,1]$ to several intervals by a polynomial transformation.

LEMMA 1. Let $T$ be a polynomial of degree $N \geq 2$ with real and simple zeros $x_{1}<x_{2}<\cdots<x_{N}$. Let $y_{1}<y_{2}<\cdots<y_{N-1}$ be the zeros of $T^{\prime}$. If $\left|T\left(y_{j}\right)\right| \geq$ $1(j=1,2, \ldots, N-1)$, then there exists a unique sequence of closed intervals $\left\{E_{1}, E_{2}, \ldots, E_{N}\right\}$ such that
(a) $T\left(E_{i}\right)=[-1,1]$,
(b) $x_{i} \in E_{i}$,
(c) $E_{i} \cap E_{i+1}$ contains at most one point $(i=1, \ldots, N-1)$.

PROOF. The statement is obvious and can most easily be verified from Figure 1. The set $E_{i} \cap E_{i+1}$ will contain one point if and only if $T\left(y_{i}\right)= \pm 1$.

In the remainder of this paper we suppose that $T$ satisfies the conditions given in Lemma 1. Following Bessis-Moussa [8], Moussa [20], and Bessis-Geronimo-Moussa [7] we now construct a measure $\mu$ on $T^{-1}\left(E_{0}\right)$ starting from a given measure $\mu_{0}$ on $E_{0}$. Let $W$ be a polynomial of degree $N-1$ such that

$$
\begin{gathered}
\frac{W(z)}{T(z)-x}=\sum_{i=1}^{N} \frac{w_{i}(x)}{z-T_{i}^{-1}(x)}, \quad x \in E_{0}, \quad z \in \mathbf{C} \backslash E \\
w_{i}(x)>0, \quad x \in E_{0}, \quad i=1,2, \ldots, N, \\
\sum_{i=1}^{N} w_{i}(x)=1, \quad x \in E_{0},
\end{gathered}
$$

where $\left\{T_{i}^{-1}: i=1,2, \ldots, N\right\}$ is a complete set of inverse branches of $T$ with $T_{i}^{-1}([-1,1])=E_{i}$. There is always at least one possible choice, namely $W=T^{\prime} / N$, in which case $w_{i}(x)=1 / N(i=1,2, \ldots, N)$. From $(2.1)$ we find

$$
w_{i}(x)=\frac{W\left(T_{i}^{-1}(x)\right)}{T^{\prime}\left(T_{i}^{-1}(x)\right)}, \quad i=1,2, \ldots, N
$$




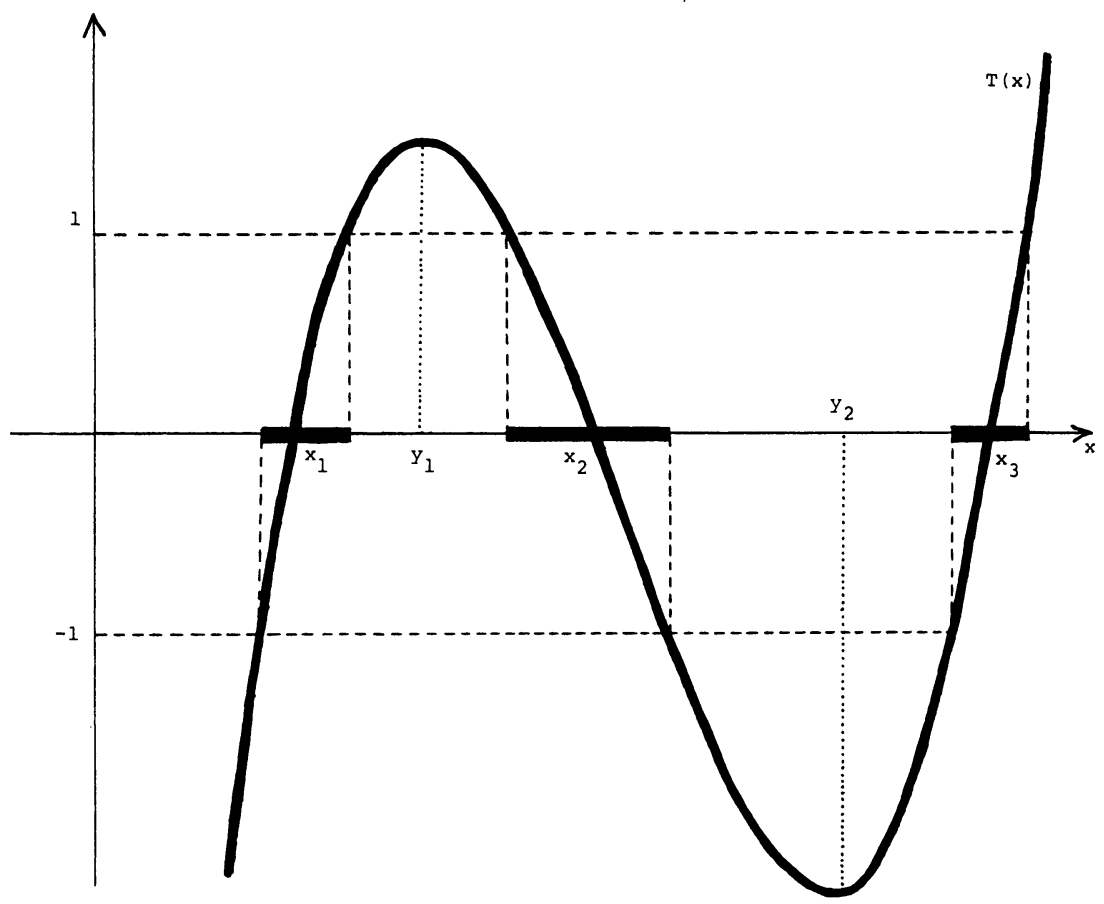

FIgURE 1. The set $E=T^{-1}([-1,1])$ are the intervals indicated by bold lines

which means that (2.2) is satisfied if and only if $W$ has the same sign as $T^{\prime}$ on every $E_{i}(i=1,2, \ldots, N)$. In particular, this implies that the zeros $z_{1}<z_{2}<\cdots<$ $z_{N-1}$ of $W$ are such that $z_{i}$ is between the intervals $E_{i}$ and $E_{i+1}$, or possibly at the endpoints of these intervals. We construct a measure $\mu$ on $E=T^{-1}\left(E_{0}\right)$ by imposing that for every Borel set $A$ in $E_{0}$

$$
\mu\left(T_{i}^{-1}(A)\right)=\int_{A} w_{i}(x) d \mu_{0}(x)
$$

From this it follows that for every $f \in L_{1}(\mu)$

$$
\int_{E} f(x) d \mu=\sum_{i=1}^{N} \int_{E_{0}} w_{i}(x) f\left(T_{i}^{-1}(x)\right) d \mu_{0}(x) .
$$

REMARK 1. From (2.2) and (2.5) we see that the absolutely continuous, singular continuous, and discrete parts of $\mu_{0}$ are mapped into, respectively, the absolutely continuous, singular continuous, and discrete parts of $\mu$. Consequently, if $\sigma_{0}$ and $\sigma$ are, respectively, the absolutely continuous parts of $\mu_{0}$ and $\mu$ then

$$
\sigma(x)=|W(x)| \sigma_{0}(T(x)) .
$$

REMARK 2. The Stieltjes transform of a measure $\mu$ is defined to be

$$
S(z ; \mu)=\int \frac{d \mu(x)}{z-x}, \quad z \notin \operatorname{supp}(\mu) .
$$


Setting $f(x)=1 /(z-x)(z \notin E)$ in (2.5) gives the following relation among Stieltjes transforms:

$$
S(z ; \mu)=W(z) S\left(T(z), \mu_{0}\right)
$$

Let $\left\{p_{n}(x ; \mu): n=0,1,2, \ldots\right\}$ be the orthonormal polynomials with orthogonality measure $\mu$ on $E$, i.e.,

$$
\int_{E} p_{n}(x ; \mu) p_{m}(x ; \mu) d \mu(x)=\delta_{n, m}, \quad n, m \geq 0 .
$$

Then there is an easy relation between $p_{n N}(x ; \mu)$ and $p_{n}\left(x ; \mu_{0}\right)$.

THEOREM $1[\mathbf{7}, 8]$. Suppose $\mu_{0}$ is a measure on $E_{0} \subset[-1,1]$ and $\mu$ is the measure obtained from $\mu_{0}$ by (2.5). Then

$$
p_{n N}(x ; \mu)=p_{n}\left(T(x) ; \mu_{0}\right), \quad n=0,1,2, \ldots .
$$

PROOF. We will show that

$$
\int_{E} x^{m} p_{n}(T(x) ; \mu) d \mu(x)=0, \quad m<n N,
$$

and

$$
\int_{E} p_{n}^{2}\left(T(x) ; \mu_{0}\right) d \mu(x)=1
$$

To prove (2.9) we note that from (2.5)

$$
\begin{aligned}
\int_{E} x^{m} & p_{n}\left(T(x) ; \mu_{0}\right) d \mu(x) \\
& =\sum_{i=1}^{N} \int_{E_{0}} w_{i}(x)\left(T_{i}^{-1}(x)\right)^{m} p_{n}\left(x ; \mu_{0}\right) d \mu_{0}(x) .
\end{aligned}
$$

If we expand (2.1) into powers of $1 / z$, then the left-hand side of $(2.1)$ becomes

$$
\sum_{n=0}^{\infty} \frac{1}{z^{n+1}} \sum_{i=1}^{N} w_{i}(x)\left(T_{i}^{-1}(x)\right)^{n}
$$

and the right-hand side is

$$
W(z) \sum_{n=0}^{\infty} \frac{x^{n}}{(T(z))^{n+1}}
$$

Since

$$
\frac{W(z)}{(T(z))^{n+1}}=O\left(\frac{1}{z^{n N+1}}\right)
$$

we have

$$
\frac{W(z)}{T(z)-x}=\sum_{n=0}^{\infty} \frac{1}{z^{n+1}} \Pi_{[n / N]}(x),
$$

where $\Pi_{m}$ is a polynomial of degree at most $m$ and $[n / N]$ is the integer part of $n / N$. Hence by comparing the coefficients of $z^{-m-1}$

$$
\int_{E} x^{m} p_{n}\left(T(x) ; \mu_{0}\right) d \mu(x)=\int_{E_{0}} \Pi_{[m / N]}(x) p_{n}\left(x ; \mu_{0}\right) d \mu_{0}(x)
$$


and the latter integral is zero for $m<n N$. For (2.10) we have by (2.5)

$$
\int_{E} p_{n}^{2}\left(T(x) ; \mu_{0}\right) d \mu=\sum_{i=1}^{N} \int_{E_{0}} w_{i}(x) p_{n}^{2}\left(x ; \mu_{0}\right) d \mu_{0}(x)
$$

and this is equal to one by $(2.2)$ and the orthonormality of the $\left\{p_{n}\left(x ; \mu_{0}\right)\right\}$.

A sequence $\left\{p_{n}(x): n=0,1,2, \ldots\right\}$ of orthogonal polynomials on the real line satisfies a three-term recurrence formula

$$
x p_{n}(x)=a_{n+1} p_{n+1}(x)+b_{n} p_{n}(x)+a_{n} p_{n-1}(x), \quad n=0,1, \ldots,
$$

with $p_{0}(x)=1, p_{-1}(x)=0, a_{n}>0$, and $b_{n} \in \mathbf{R}$. The $k$ th associated polynomials $\left\{p_{n}^{(k)}(x), n=0,1,2, \ldots\right\}(k \in \mathrm{N})$ satisfy the "shifted recurrence relation"

$$
x p_{n}^{(k)}(x)=a_{n+k+1} p_{n+1}^{(k)}(x)+b_{n+k} p_{n}^{(k)}(x)+a_{n+k} p_{n-1}^{(k)}(x), \quad n=0,1,2, \ldots,
$$

with $p_{0}^{(k)}=1$ and $p_{-1}^{(k)}(x)=0$. Let $\left\{a_{n+1}^{0}, b_{n}^{0}: n=0,1,2, \ldots\right\}$ be the recurrence coefficients for $\left\{p_{n}\left(x ; \mu_{0}\right)\right\}$ and $\left\{a_{n+1}, b_{n}: n=0,1,2, \ldots\right\}$ be those for $\left\{p_{n}(x ; \mu)\right\}$. Then we have

THEOREM 2. With the same notation as in Theorem 1, we have

$$
p_{n N-1}^{(1)}(x ; \mu)=\frac{a_{1}}{a_{1}^{0}} W(x) p_{n-1}^{(1)}\left(T(x) ; \mu_{0}\right), \quad n=0,1,2, \ldots
$$

PROOF. It is well known (and one can easily check) that

$$
p_{n-1}^{(1)}(x ; \mu)=a_{1} \int_{E} \frac{p_{n}(x ; \mu)-p_{n}(y ; \mu)}{x-y} d \mu(y) .
$$

By (2.5) and (2.8) we have

$$
\begin{aligned}
p_{n N-1}^{(1)}(x ; \mu) & =a_{1} \int_{E} \frac{p_{n}\left(T(x) ; \mu_{0}\right)-p_{n}\left(T(y) ; \mu_{0}\right)}{x-y} d \mu(y) \\
& =a_{1} \sum_{i=1}^{N} \int_{E_{0}} w_{i}(y) \frac{p_{n}\left(T(x) ; \mu_{0}\right)-p_{n}\left(y ; \mu_{0}\right)}{x-T_{i}^{-1}(y)} d \mu_{0}(y) .
\end{aligned}
$$

Using (2.1) gives

$$
p_{n N-1}^{(1)}(x ; \mu)=a_{1} W(x) \int_{E} \frac{p_{n}\left(T(x) ; \mu_{0}\right)-p_{n}\left(y ; \mu_{0}\right)}{T(x)-y} d \mu_{0}(y)
$$

from which the desired result follows.

Let $\mu^{(1)}$ and $\mu_{0}^{(1)}$ be the orthogonality measures associated with $\left\{p_{n}^{(1)}(x ; \mu)\right\}$ and $\left\{p_{n}^{(1)}\left(x ; \mu_{0}\right)\right\}$ respectively. The next theorem describes how the Stieltjes transforms associated with $\mu^{(1)}$ and $\mu_{0}^{(1)}$ are related and gives a relation analogous to (2.5) among these two measures. A result similar to $(2.13)$ when $W(z)=T^{\prime}(z) / N$ was given by Barnsley, Geronimo, and Harrington [4].

THEOREM 3. Let $S\left(z, \mu^{(1)}\right)$ and $S\left(z, \mu_{0}^{(1)}\right)$ be the Stieltjes transforms associated with $\mu^{(1)}$ and $\mu_{0}^{(1)}$ respectively. Then

$$
S\left(z ; \mu^{(1)}\right)=\left(\frac{a_{1}^{0}}{a_{1}}\right)^{2} \frac{S\left(T(z) ; \mu_{0}^{(1)}\right)}{W(z)}+\frac{\hat{p}_{N-2}^{(2)}(z ; \mu)}{\hat{p}_{N-1}^{(1)}(z ; \mu)},
$$


where $\hat{p}_{N-2}^{(2)}$ and $\hat{p}_{N-1}^{(1)}$ are monic orthogonal polynomials. Let $\left\{z_{i}, i=1,2, \ldots, N-\right.$ $1\}$ be the zeros of $W(z)$ and suppose that $S\left(T\left(z_{i}\right), \mu_{0}^{(1)}\right)$ exists. Then (2.13) implies that for every bounded continuous function $f$,

$$
\begin{aligned}
\int f(x) d \mu^{(1)}(x)= & \left(\frac{a_{1}^{0}}{a_{1}}\right)^{2} \sum_{i=1}^{N} \int \frac{w_{i}(x)}{\left|W\left(T_{i}^{-1}(x)\right)\right|^{2}} f\left(T_{i}^{-1}(x)\right) d \mu_{0}^{(1)}(x) \\
& +\sum_{i=1}^{N-1}\left\{\left(\frac{a_{1}^{0}}{a_{1}}\right)^{2} S\left(T\left(z_{i}\right) ; \mu_{0}^{(1)}\right)+c \hat{p}_{N-2}^{(2)}\left(z_{i} ; \mu\right)\right\} \frac{f\left(z_{i}\right)}{W^{\prime}\left(z_{i}\right)},
\end{aligned}
$$

where $c$ is the leading coefficient of $T$ and $W$ (see (3.6)).

ProOF. A well-known relation between $S(z ; \mu)$ and $S\left(z ; \mu^{(1)}\right)$ is given by

$$
S(z ; \mu)=\frac{1}{z-b_{0}-a_{1}^{2} S\left(z ; \mu^{(1)}\right)} .
$$

If we use this relation for $\mu_{0}$ and $\mu_{0}^{(1)}$ with $z$ replaced by $T(z)$, we find

$$
S\left(T(z) ; \mu_{0}\right)=\frac{1}{T(z)-b_{0}^{0}-\left(a_{1}^{0}\right)^{2} S\left(T(z), \mu_{0}^{(1)}\right)} .
$$

Multiply both sides of this equation by $W(z)$. Then by $(2.7)$

$$
S(z ; \mu)=\frac{W(z)}{T(z)-b_{0}^{0}-\left(a_{1}^{0}\right)^{2} S\left(T(z), \mu_{0}^{(1)}\right)} .
$$

Substituting this result into (2.16) and then solving the resulting equation for $S\left(z, \mu^{(1)}\right)$ yields

$$
S\left(z, \mu^{(1)}\right)=\left(\frac{a_{1}^{0}}{a_{1}}\right)^{2} \frac{S\left(T(z) ; \mu_{0}^{(1)}\right)}{W(z)}+\frac{\left(z-b_{0}\right) W(z)-T(z)+b_{0}^{0}}{a_{1}^{2} W(z)} .
$$

From (2.12) with $n=1$ and the definition of $W(z)$ we have that $\hat{p}_{N-1}^{(1)}(z)=W(z) / c$. Furthermore (2.8) with $n=1$ tells us that

$$
\hat{p}_{N}(z)=\frac{T(z)-b_{0}^{0}}{c} .
$$

The result now follows from the fact that $a_{1}^{2} \hat{p}_{N-2}^{(2)}(z)=\left(z-b_{0}\right) \hat{p}_{N-1}^{(1)}(z)-\hat{p}_{N}(z)$ (see also (3.10)). To prove (2.14) it is sufficient to take $f(x)=1 /(z-x)$. Utilizing the fact that

$$
\begin{aligned}
\frac{1}{W(z)} \frac{1}{T(z)-x}= & \sum_{i=1}^{N} \frac{1}{W\left(T_{i}^{-1}(x)\right)} \frac{1}{T^{\prime}\left(T_{i}^{-1}(x)\right)} \frac{1}{z-T_{i}^{-1}(x)} \\
& +\sum_{i=1}^{N-1} \frac{1}{W^{\prime}\left(z_{i}\right)} \frac{1}{T\left(z_{i}\right)-x} \frac{1}{z-z_{i}}
\end{aligned}
$$

and (2.4), in (2.13) gives (2.14) for $f(x)=1 /(z-x)$.

REMARK 3 . We note that if one wishes to start with a measure $\mu_{0}^{(1)}$ one must check that supp $\mu_{0}$ is contained in $[-1,1]$. (2.13) shows that mass points at the 
zeros of $W(z)$ may appear in the measure $\mu^{(1)}$. Letting $\sigma^{(1)}$ be the absolutely continuous part of $\mu^{(1)}$ and $\sigma_{0}^{(1)}$ be the absolutely continuous part of $\mu_{0}^{(1)}$, we see that

$$
\sigma^{(1)}(x)=\left(\frac{a_{1}^{0}}{a_{1}}\right)^{2} \frac{1}{|W(x)|} \sigma_{0}^{(1)}(T(x)), \quad x \in T^{-1}(-1,1) .
$$

From (2.8) we see that there is a simple relation between the zeros of $p_{n N}(x ; \mu)$ and $p_{n}\left(x ; \mu_{0}\right)$. Let $\left\{x_{i, n}^{0}, i=1,2, \ldots, n\right\}$ denote the zeros of $p_{n}\left(x ; \mu_{0}\right)$. Then the zeros $\left\{x_{i, j, n N}, i=1,2, \ldots, N, j=1,2, \ldots, n\right\}$ of $p_{n N}(x ; \mu)$ are given by

$$
x_{i, j, n N}=T_{i}^{-1}\left(x_{j, n}^{0}\right), \quad 1 \leq j \leq n, 1 \leq i \leq N .
$$

Recall that the Christoffel numbers $\left\{\lambda_{j, n}: 1 \leq j \leq n, n=1,2, \ldots\right\}$ for a system of orthogonal polynomials $\left\{p_{n}(x)\right\}$ with spectral measure $\mu$ are the Côtes numbers for the Gauss-Jacobi quadrature

$$
\int \pi(x) d \mu(x)=\sum_{j=1}^{n} \lambda_{j, n} \pi\left(x_{j, n}\right)
$$

which holds for every polynomial $\pi$ of degree at most $2 n-1$. These Christoffel numbers are also equal to the residues of

$$
\frac{\hat{p}_{n-1}^{(1)}(x)}{\hat{p}_{n}(x)}=\sum_{j=1}^{n} \frac{\lambda_{j, n}}{x-x_{j, n}} .
$$

THEOREM 4. Let $\left\{\lambda_{j, n}^{0}\right\}$ be the Christoffel numbers for the spectral mesure $\mu_{0}$ on $E_{0}$ and $\left\{\lambda_{j, n}\right\}$ those for the measure $\mu$ on $E$. Then

$$
\lambda_{i, j, n N}=\lambda_{j, n}^{0} w_{i}\left(x_{j, n}^{0}\right), \quad 1 \leq j \leq n, 1 \leq i \leq N .
$$

PrOOF. From (2.8) and (2.12) we see that the $[(n N-1) / n N]$ Padé approximant to $S(z, \mu)$ is

$$
\frac{\hat{p}_{n N-1}^{(1)}(x ; \mu)}{\hat{p}_{n N}(x ; \mu)}=W(x) \frac{\hat{p}_{n-1}^{(1)}\left(T(x) ; \mu_{0}\right)}{\hat{p}_{n}\left(T(x) ; \mu_{0}\right)},
$$

where $\hat{p}_{i}$ denotes the monic orthogonal polynomial of degree $i$. Evaluating the residues of the above rational fractions and using the relation between the zeros of $\hat{p}_{n N}(x ; \mu)$ and $\hat{p}_{n}\left(x ; \mu_{0}\right)$ given above gives the result.

If one uses Chebyshev polynomials of the first kind on $[-1,1]$, then it is well known that $\lambda_{j, n}^{0}=1 / n(j=1, \ldots, n)$. Choosing $W(x)=T^{\prime}(x) / N$ gives Christoffel numbers $\lambda_{j, n N}=1 / n N$ and the Gauss-Jacobi quadrature uses equal weights under these conditions.

III. Jacobi matrices. Let $\left\{p_{n}(x): n=0,1,2, \ldots\right\}$ be a sequence of orthogonal polynomials that satisfy the recurrence relation (2.11); then closely related to these polynomials is the Jacobi matrix

$$
J=\left(\begin{array}{ccccc}
b_{0} & a_{1} & & & 0 \\
a_{1} & b_{1} & a_{2} & & \\
0 & a_{2} & b_{2} & a_{3} & \\
& & \ddots & \ddots & \ddots
\end{array}\right) .
$$


Let $l_{2}$ be the square summable sequences $\left\{\psi=(\psi(0), \psi(1), \psi(2), \ldots): \sum_{n=0}^{\infty} \psi^{2}(n)\right.$ $<\infty\}$. Then $J$ acts as an operator from $l_{2}$ to $l_{2}$. There is an easy relationship between the resolvents of the Jacobi matrices of $\left\{p_{n}\left(x ; \mu_{0}\right)\right\}$ and $\left\{p_{n}(x ; \mu)\right\}$ :

THEOREM $5[6]$. Let $\left\{a_{n+1}^{0}, b_{n}^{0}: n=0,1,2, \ldots\right\}$ be the recurrence coefficients for the measure $\mu_{0}$ on $E_{0} \subset[-1,1]$ with Jacobi matrix $J_{0}$ and $\left\{a_{n+1}, b_{n}: n=\right.$ $0,1,2, \ldots\}$ the recurrence coefficients for $\mu$ on $E=T^{-1}\left(E_{0}\right)$ with Jacobi matrix $J$. Then

$$
W(z)\left(T(z)-J_{0}\right)^{-1}=D(z-J)^{-1} D^{*}, \quad z \notin E,
$$

where $D: l_{2} \rightarrow l_{2}$ acts as $(D \psi)(n)=\psi(n N)$ and $D^{*}$ is the adjoint (transpose) of $D$.

PROOF. The $(n, m)$-entry of $(z-J)^{-1}$ is given by

$$
\left[(z-J)^{-1}\right]_{n, m}=\int_{E} \frac{p_{n}(x ; \mu) p_{m}(x ; \mu)}{z-x} d \mu(x)
$$

(Stone [24, p. 546]). By (2.8) we then have

$$
\begin{aligned}
{\left[D(z-J)^{-1} D^{*}\right]_{n, m} } & =\left[(z-J)^{-1}\right]_{n N, m N} \\
& =\int_{E} \frac{p_{n}\left(T(x) ; \mu_{0}\right) p_{m}\left(T(x) ; \mu_{0}\right)}{z-x} d \mu(x) .
\end{aligned}
$$

Next use (2.5), (2.1), and (2.8) in the integral to find

$$
\left[D(z-J)^{-1} D^{*}\right]_{n, m}=W(z) \int_{E_{0}} \frac{p_{n}\left(x ; \mu_{0}\right) p_{m}\left(x ; \mu_{0}\right)}{T(z)-x} d \mu_{0}(x),
$$

and the last integral is the $(n, m)$-entry of $\left(T(z)-J_{0}\right)^{-1}$.

The resolvent formula (3.1) uniquely determines the recurrence coefficients $\left\{a_{n+1}, b_{n}: n=0,1,2, \ldots\right\}$ when $\left\{a_{n+1}^{0}, b_{n}^{0}: n=0,1,2, \ldots\right\}$ are given. This follows by looking at the $(0,0)$-entries in $(3.1)$.

If one wants to solve (3.1) for $J$, then one runs into problems since $D$ is not invertible. In order to get around that problem we introduce the projections

$$
P=D^{*} D, \quad Q=I-P .
$$

Following Bellissard [6], we arrive at the following Schur decomposition.

LEMMA 2. Let $\tilde{J}=Q J Q$. Then for $z \in \mathbf{C} \backslash(E \cup\{0\})$

$$
D(z-J)^{-1} D^{*}=\left[z-D J D^{*}-D J Q(z-\tilde{J})^{-1} Q J D^{*}\right]^{-1} .
$$

PROOF. We will show that

$$
D(z-J)^{-1} D^{*}\left[z-D J D^{*}-D J Q(z-\tilde{J})^{-1} Q J D^{*}\right]=I .
$$

The left-hand side of this equation is equal to

$$
z D(z-J)^{-1} D^{*}-D(z-J)^{-1} P J D^{*}-D(z-J)^{-1} P J Q(z-\tilde{J})^{-1} Q J D^{*}
$$

By the definition of $Q$

$$
\tilde{J}_{m, n}=[Q J Q]_{m, n}=0, \quad m=0(\bmod N) \text { or } n=0(\bmod N)
$$


so that

$$
\left[(z-\tilde{J})^{-1}\right]_{m N, n}=0, \quad n \neq n M
$$

We then easily find

$$
P(z-\tilde{J})^{-1} Q=0 .
$$

Using this we can rewrite the last term in (3.4) as

$$
D(z-J)^{-1}[P J Q+P z](z-\tilde{J}) Q J D^{*} .
$$

Since $P=I-Q$ and $D Q=0$ this is equal to

$$
D(z-J)^{-1}[(z-\tilde{J})-(z-J) Q](z-\tilde{J})^{-1} Q J D^{*}=D(z-J)^{-1} Q J D^{*} .
$$

Therefore (3.4) becomes

$$
\begin{gathered}
z D(z-J)^{-1} D^{*}-D(z-J)^{-1} P J D^{*}-D(z-J)^{-1} Q J D^{*} \\
=D(z-J)^{-1}[z-P J-Q J] D^{*}=D D^{*}
\end{gathered}
$$

and since $D D^{*}=I$ this proves the result.

A combination of (3.1) and (3.3) gives

$$
T(z)-J_{0}=W(z)\left[z-D J D^{*}-D J Q(z-\tilde{J})^{-1} Q J D^{*}\right] .
$$

This equation then leads to

THEOREM 6 [8]. Let

$$
\begin{aligned}
T(z) & =c z^{N}+\alpha_{1} z^{N-1}+\alpha_{2} z^{N-2}+\cdots+\alpha_{N} \\
W(z) & =c z^{N-1}+\beta_{1} z^{N-2}+\cdots+\beta_{N-1} .
\end{aligned}
$$

Then

$$
\begin{gathered}
b_{n N}=\frac{\beta_{1}-\alpha_{1}}{c}, \quad n=0,1,2, \ldots, \\
\prod_{i=1}^{N} a_{n N+i}=\frac{1}{c} a_{n+1}^{0}, \quad n=0,1,2, \ldots, \\
\hat{p}_{N-1}^{(n N+1)}(z ; \mu)=\frac{1}{c} W(z), \quad n=0,1,2, \ldots, \\
a_{n N}^{2} \hat{p}_{N-2}^{(n N-N+1)}(z ; \mu)+a_{n N+1}^{2} \hat{p}_{N-2}^{(n N+2)}(z ; \mu) \\
=\frac{1}{c}\left\{b_{n}^{0}-T(z)+\left(z-\frac{\beta_{1}-\alpha_{1}}{c}\right) W(z)\right\}, \quad n=0,1,2, \ldots,
\end{gathered}
$$

where $a_{0}=0$ and $\hat{p}_{n}^{(k)}(z ; \mu)$ is the monic associated polynomial of order $k$ (i.e., with leading coefficient one).

PROOF. If one compares the coefficients of $z^{N-1}$ in (3.5), then

$$
\left(\beta_{1}-\alpha_{1}\right) I=c D J D^{*}
$$

and since

$$
\left[D J D^{*}\right]_{n, n}=J_{n N, n N}=b_{n N}
$$


this gives (3.7). From (3.5) we also find, using (3.11),

$$
W(z)\left[D J Q(z-\tilde{J})^{-1} Q J D^{*}\right]_{n, n+1}=a_{n+1}^{0} \text {. }
$$

Let $J^{(n)}(n=0,1,2, \ldots)$ be a $(N-1) \times(N-1)$ Jacobi matrix defined by

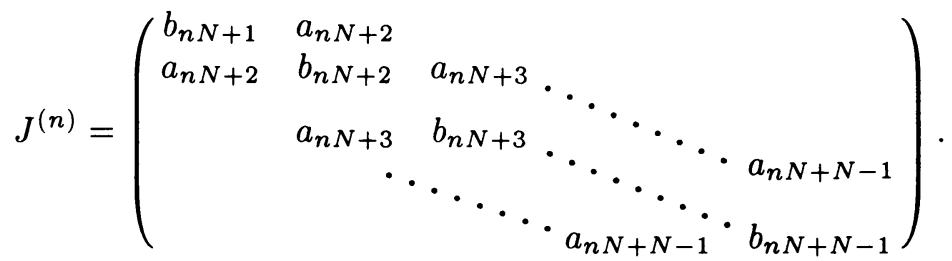

Then $\tilde{J}$ is a block diagonal matrix with the $J^{(n)}$ matrices on the diagonal separated in the $(n N, n N)$-entries by zeros:

$$
\tilde{J}=\left(\begin{array}{cccccccc}
0 & & & & & & & \\
& J^{(0)} & & & & & & \\
& & 0 & & & & & \\
& & & J^{(1)} & & & & \\
& & & 0 & & & \\
& & & & J^{(2)} & & \\
& & & & & 0 & \\
& & & & & & & \ddots
\end{array}\right)
$$

and therefore

$$
(z-\tilde{J})^{-1}=\left(\begin{array}{ccccc}
1 / z & & & & \\
& \left(z-J^{(0)}\right)^{-1} & & & \\
& 1 / z & & \\
& & \left(z-J^{(1)}\right)^{-1} & & \\
& & & 1 / z & \\
& & & & \ddots
\end{array}\right) .
$$

Some simple algebraic manipulations show that

$$
\begin{aligned}
{\left[D J Q(z-\tilde{J})^{-1} Q J D^{*}\right]_{n, n+1} } & =\left[J Q(z-\tilde{J})^{-1} Q J\right]_{n N, n N+N} \\
& =a_{n N+1} a_{n N+N}\left[(z-\tilde{J})^{-1}\right]_{n N+1, n N+N-1} .
\end{aligned}
$$

Now

$$
\left[(z-\tilde{J})^{-1}\right]_{n N+1, n N+N-1}=\left[\left(z-J^{(n)}\right)^{-1}\right]_{1, N-1}=\frac{\prod_{i=2}^{N-1} a_{n N+i}}{\hat{p}_{N-1}^{(n N+1)}(z ; \mu)}
$$

the last equation follows by calculating $\left(z-J^{(n)}\right)^{-1}$ using cofactors. If we insert this in (3.12), we find

$$
W(z) \prod_{i=1}^{N} a_{n N+i} / \hat{p}_{N-1}^{(n N+1)}(z ; \mu)=a_{n+1}^{0},
$$

and this implies both (3.8) and (3.9). In a similar way we find from (3.5), using $(3.11)$,

$$
W(z)\left[D J Q(z-\tilde{J})^{-1} Q J D^{*}\right]_{n, n}=b_{n}^{0}-T(z)+\left(z-\frac{\beta_{1}-\alpha_{1}}{c}\right) W(z) .
$$


Some easy algebra gives

$$
\begin{aligned}
{\left[D J Q(z-\tilde{J})^{-1} Q J D^{*}\right]_{n, n}=} & {\left[J Q(z-\tilde{J})^{-1} Q J\right]_{n N, n N} } \\
= & a_{n N}^{2}\left[(z-\tilde{J})^{-1}\right]_{n N-1, n N-1} \\
& +a_{n N+1}^{2}\left[(z-\tilde{J})^{-1}\right]_{n N+1, n N+1}
\end{aligned}
$$

where for $n=0$ we set $a_{0}=0$. Now

$$
\begin{gathered}
{\left[(z-\tilde{J})^{-1}\right]_{n N-1, n N-1}=\left[\left(z-J^{(n-1)}\right)^{-1}\right]_{N-1, N-1}} \\
=\frac{\hat{p}_{N-2}^{(n N-N+1)}(z ; \mu)}{\hat{p}_{N-1}^{(n N-N+1)}(z ; \mu)}, \\
{\left[(z-\tilde{J})^{-1}\right]_{n N+1, n N+1}=\left[\left(z-J^{(n)}\right)^{-1}\right]_{1,1}=\frac{\hat{p}_{N-2}^{(n N+2)}(z ; \mu)}{\hat{p}_{N-1}^{(n N+1)}(z ; \mu)},}
\end{gathered}
$$

and (3.10) follows by using (3.8) and (3.9).

If one uses the formulas

$$
\left(z-\frac{\beta_{1}-\alpha_{1}}{c}\right) \frac{W(z)}{c}=\left(z-b_{n N}\right) \hat{p}_{N-1}^{(n N+1)}(z ; \mu)
$$

and

$$
\left(z-b_{n N}\right) \hat{p}_{N-1}^{(n N+1)}(z ; \mu)=\hat{p}_{N}^{(n N)}(z ; \mu)+a_{n N+1}^{2} \hat{p}_{N-2}^{(n N+2)}(z ; \mu)
$$

then (3.10) becomes

$$
\frac{T(z)-b_{n}^{0}}{c}=\hat{p}_{N}^{(n N)}(z ; \mu)-a_{n N}^{2} \hat{p}_{N-2}^{(n N-N+1)}(z ; \mu) .
$$

IV. Properties of the new orthogonal polynomials. So far we have a simple relation between the polynomials $p_{n N}(x ; \mu)$ and $p_{n}\left(x ; \mu_{0}\right)$ given by Theorem 1 , and a similar relation between $p_{n N-1}^{(1)}(x ; \mu)$ and $p_{n-1}^{(1)}\left(x ; \mu_{0}\right)$ given by Theorem 2 . A natural question then is to find a formula for $p_{n N+j}(x ; \mu)$ and $p_{n N+j-1}^{(1)}(x ; \mu)$ in terms of the initial system $p_{n}\left(x ; \mu_{0}\right)$ and $p_{n-1}^{(1)}\left(x ; \mu_{0}\right)$. We will give such formulas by using some Wronskian formulas. If $\left\{u_{n}\right\}$ and $\left\{v_{n}\right\}$ are two solutions of the recurrence relation (2.11), then their Wronskian is defined to be

$$
\begin{aligned}
W\left(u_{n}, v_{n}\right) & =a_{n+1}\left|\begin{array}{cc}
u_{n} & v_{n} \\
u_{n+1} & v_{n+1}
\end{array}\right| \\
& =a_{n+1}\left(u_{n} v_{n+1}-u_{n+1} v_{n}\right) .
\end{aligned}
$$

One can easily verify that $W\left(u_{n}, v_{n}\right)$ is independent of $n$.

THEOREM 7. Let $j$ be any integer such that $0 \leq j<N$. Then

$$
\begin{array}{r}
p_{n N+j}(x ; \mu)=\frac{1}{W(x)} \frac{a_{n+1}^{0}}{a_{n N+1}}\left\{\frac{a_{n N+1}}{a_{n N+j+1}} p_{N-j-1}^{(n N+j+1)}(x ; \mu) p_{n}\left(T(x) ; \mu_{0}\right)\right. \\
\left.+p_{j-1}^{(n N+1)}(x ; \mu) p_{n+1}\left(T(x) ; \mu_{0}\right)\right\}
\end{array}
$$


and

$$
\begin{aligned}
p_{n N+j-1}^{(1)}(x ; \mu)=\frac{a_{1}}{a_{1}^{0}} \frac{a_{n+1}^{0}}{a_{n N+1}}\left\{\frac{a_{n N+1}}{a_{n N+j+1}} p_{N-j-1}^{(n N+j+1)}(x ; \mu) p_{n-1}^{(1)}\left(T(x) ; \mu_{0}\right)\right. \\
\left.+p_{j-1}^{(n N+1)}(x ; \mu) p_{n}^{(1)}\left(T(x) ; \mu_{0}\right)\right\} .
\end{aligned}
$$

ProOF. Let $n, N$ and $j$ be fixed integers. Then the sequences $p_{(n+1) N+k}(x ; \mu)$, $p_{N+k-1}^{(n N+1)}(x ; \mu)$, and $p_{N+k-j-1}^{(n N+j+1)}(x ; \mu)$ are solutions of the recurrence relation

$$
x u_{k}=a_{n N+k+1} u_{k+1}+b_{n N+k} u_{k}+a_{n N+k} u_{k-1} .
$$

Since this is a linear recurrence relation of second order, these three solutions have to be linearly dependent and therefore there exist $A, B$, and $C$ (independent of $k$ ) such that

$$
A p_{N+k-1}^{(n N+1)}(x ; \mu)=B p_{N+k-j-1}^{(n N+j+1)}(x ; \mu)+C p_{(n+1) N+k}(x ; \mu) .
$$

Setting $k$ equal to $j-N$ gives

$$
A p_{j-1}^{(n N+1)}(x ; \mu)=C p_{n N+j}(x ; \mu),
$$

and setting $k$ equal to $j-N+1$ gives

$$
A p_{j}^{(n N+1)}(x ; \mu)=B+C p_{n N+j+1}(x ; \mu) .
$$

Therefore

$$
C=A \frac{p_{j-1}^{(n N+1)}(x ; \mu)}{p_{n N+j}(x ; \mu)}
$$

and

$$
\begin{aligned}
B & =\frac{A}{p_{n N+j}(x ; \mu)}\left\{p_{j}^{(n N+1)}(x ; \mu) p_{n N+j}(x ; \mu)-p_{j-1}^{(n N+1)}(x ; \mu) p_{n N+j+1}(x ; \mu)\right\} \\
& =\frac{A}{p_{n N+j}(x ; \mu)} \frac{1}{a_{n N+j+1}} W\left(p_{n N+k}, p_{k-1}^{(n N+1)}\right) .
\end{aligned}
$$

We can evaluate the Wronskian by choosing $k=0$, giving

$$
W\left(p_{n N+k}, p_{k-1}^{(n N+1)}\right)=a_{n N+1} p_{n N}(x ; \mu)
$$

so that (4.3) becomes

$$
\begin{aligned}
p_{n N+j}(x ; \mu) p_{N+k-1}^{(n N+1)}(x ; \mu)= & \frac{a_{n N+1}}{a_{n N+j+1}} p_{N+k-j-1}^{(n N+j+1)}(x ; \mu) p_{n N}(x ; \mu) \\
& +p_{j-1}^{(n N+1)}(x ; \mu) p_{(n+1) N+k}(x ; \mu) .
\end{aligned}
$$

Let $k=0$ in this formula and use (3.8) and (3.9); then (4.1) follows. Similar reasoning using the solutions $p_{(n+1) N+k-1}^{(1)}(x ; \mu), p_{N+k-1}^{(n N+1)}(x ; \mu)$, and $p_{N+k-j-1}^{(n N+j-1)}(x ; \mu)$ will lead to (4.2).

The next theorem shows us how to relate $a_{n N}$ to $a_{n}^{0}$. 
THEOREM 8. Let $\left(\mathbf{z}^{j}\right)^{t}=\left(z_{1}^{j}, z_{2}^{j}, \ldots, z_{N-1}^{j}\right)$, where $\left\{z_{i}: i=1,2, \ldots, N-1\right\}$ are the zeros of $W(z)$ and let $\left(\gamma_{n}\right)^{t}=\left(\gamma_{1, n}, \gamma_{2, n}, \ldots, \gamma_{N-1, n}\right)$, where

$$
\gamma_{i, n}=\frac{-a_{n+1}^{0}}{c} \frac{p_{n}\left(T\left(z_{i}\right) ; \mu_{0}\right)}{p_{n+1}\left(T\left(z_{i}\right) ; \mu_{0}\right)}, \quad i=1,2, \ldots, N-1 .
$$

Then

$$
a_{(n+1) N}^{2}=\frac{\operatorname{det}\left[1, \mathbf{z}, \mathbf{z}^{2}, \ldots, \gamma_{n}\right]}{\operatorname{det}\left[1, \mathbf{z}, \mathbf{z}^{2}, \ldots, \mathbf{z}^{N-2}\right]}
$$

and

$$
\hat{p}_{N-2}^{(n N+1)}(z ; \mu)=z^{N-2}+\sum_{i=0}^{N-3} c_{i} z^{i}
$$

where

$$
c_{i}=\frac{\operatorname{det}\left[1, \mathbf{z}, \mathbf{z}^{2}, \ldots, \mathbf{z}^{i-1}, \boldsymbol{\gamma}_{n}, \mathbf{z}^{i+1}, \ldots, \mathbf{z}^{N-2}\right]}{\operatorname{det}\left[1, \mathbf{z}, \mathbf{z}^{2}, \ldots, \mathbf{z}^{N-3}, \gamma_{n}\right]}, \quad i=0,1,2, \ldots, N-3
$$

Furthermore this allows us to calculate $\left\{a_{n N+i}: i=1,2, \ldots, N-1\right\}$ and $\left\{b_{n N+i}: i=\right.$ $1,2, \ldots, N-1\}$ in terms of ratios $p_{n}\left(z ; \mu_{0}\right) / p_{n+1}\left(z ; \mu_{0}\right)$.

Proof. Consider the Wronskian (4.4) for $k=N-1$. Then

$$
\begin{aligned}
a_{n N+1} p_{n N}(x ; \mu)=a_{(n+1) N}\{ & p_{(n+1) N-1}(x ; \mu) p_{N-1}^{(n N+1)}(x ; \mu) \\
& \left.-p_{(n+1) N}(x ; \mu) p_{N-2}^{(n N+1)}(x ; \mu)\right\} .
\end{aligned}
$$

If we evaluate the above Wronskian at a zero $z_{i}$ of $W(z)$ and use (3.9), we obtain

$$
a_{n N+1} p_{n N}\left(z_{i} ; \mu\right)=-a_{(n+1) N} p_{N-2}^{(n N+1)}\left(z_{i} ; \mu\right) p_{(n+1) N}\left(z_{i} ; \mu\right) .
$$

The fact that

$$
\begin{aligned}
p_{N-1}^{(n N+1)}(z ; \mu) & =\frac{1}{a_{n N+2} a_{n N+3} \cdots a_{n N+N-1}} \hat{p}_{N-2}^{(n N+1)}(z ; \mu) \\
& =c \frac{a_{n N+1} a_{(n+1) N}}{a_{n+1}^{0}} \hat{p}_{N-2}^{(n N+1)}(z ; \mu)
\end{aligned}
$$

(see (3.8)) and Theorem 1 give

$$
a_{(n+1) N}^{2} \hat{p}_{N-2}^{(n N+1)}\left(z_{i} ; \mu\right)=\frac{-a_{n+1}^{0}}{c} \frac{p_{n}\left(T\left(z_{i}\right) ; \mu_{0}\right)}{p_{n+1}\left(T\left(z_{i}\right) ; \mu_{0}\right)}=\gamma_{i, n}, \quad i=1,2, \ldots, N-1 .
$$

If we write

$$
a_{(n+1) N}^{2} \hat{p}_{N-2}^{(n N+1)}(z ; \mu)=\sum_{i=0}^{N-2} k_{i} z^{i}
$$

and then solve for $k_{i}$ using Cramer's rule, we find

$$
k_{i}=\frac{\operatorname{det}\left[1, \mathbf{z}, \ldots, \mathbf{z}^{i-1}, \gamma_{n}, \mathbf{z}^{i+1}, \ldots, \mathbf{z}^{N-2}\right]}{\operatorname{det}\left[1, \mathbf{z}, \mathbf{z}^{2}, \ldots, \mathbf{z}^{N-2}\right]}
$$

From this we find (4.6) when $i=N-1$ and (4.7) since $k_{i}=a_{(n+1) N}^{2} c_{i}$.

To show the last assertion we write

$$
\hat{p}_{i}^{(n N+1)}(z)=Q_{i}(z)=z^{i}+\sum_{j=0}^{i-1} q_{i, j} z^{j}, \quad i=1,2, \ldots, N-2 .
$$


Since

$$
a_{n N+i-1}^{2} Q_{i-3}(z)=\left(z-b_{n N+i-1}\right) Q_{i-2}(z)-Q_{i-1}(z), \quad i=3, \ldots, N,
$$

we have the following formulas which follow by equating like powers of $z$ :

$$
b_{n N+i-1}=-q_{i-1, i-2}+q_{i-2, i-3},
$$

$$
a_{n N+i-1}^{2}=q_{i-2, i-4}-q_{i-1, i-3}-b_{n N+i-1} q_{i-2, i-3},
$$

and

$$
q_{i-3, j}=\frac{q_{i-2, j-1}-q_{i-1, j}-b_{n N+i-1} q_{i-2, j}}{a_{n N+i-1}^{2}}, \quad j=0,1, \ldots, i-2 .
$$

Since $q_{N-1, j}$ and $q_{N-2, j}$ are known, (4.10) gives us $b_{n N+N-1}$ from which follows $a_{n N+N-1}^{2}$ by (4.11). We then calculate $q_{N-3, j}$. Continuing this procedure reducing $N$ by one each time gives the result.

REMARK 4. The denominator in (4.6) is a Vandermonde determinant which for some classical polynomials is also referred to as a discriminant. Explicit formulas are available for classical polynomials (Szegö [25, p. 143]).

Let $\phi(T, W)$ denote the mapping that maps polynomials orthonormal on $E_{0}$ to polynomials orthonormal on $E$ given by (2.5) or (3.1). The above theorem allows us to determine some classes of polynomials that are mapped into each other under $\phi$.

A system of orthonormal polynomials $\left\{p_{n}(x)\right\}$ with orthogonality set $\Delta$ is in $M_{k}(\Delta)$ iff there exists $\left\{\tilde{a}_{n}\right\}_{n=1}^{\infty}$ and $\left\{\tilde{b}_{n}\right\}_{n=0}^{\infty}$ with $\tilde{a}_{n+k}=\tilde{a}_{n}>0$, and $\tilde{b}_{n+k}=\tilde{b}_{n}$ real and

$$
\lim _{n \rightarrow \infty}\left|a_{n}-\tilde{a}_{n}\right|=0 \text { and } \lim _{n \rightarrow \infty}\left|b_{n}-\tilde{b}_{n}\right|=0
$$

where the $\left\{a_{n}\right\}$ and $\left\{b_{n}\right\}$ are the coefficients in (2.11). A system of orthonormal polynomials $\left\{p_{n}(x)\right\}$ with orthogonality set $\Delta$ is in $M_{k}^{i}(\Delta) \subset M_{k}(\Delta)(i=0,1)$ iff

$$
\sum_{n=1}^{\infty} n^{i}\left\{\left|a_{n}-\tilde{a}_{n}\right|+\left|b_{n-1}-\tilde{b}_{n-1}\right|\right\}<\infty \quad(i=0,1) .
$$

THEOREM 9. Suppose the zeros of $W(z)$ are not in $E=T^{-1}\left(E_{0}\right)$ and suppose $E_{0}$ supports a class $M_{k}$ for some $k$. Then

$$
\phi(T, W): M_{k}\left(E_{0}\right) \rightarrow M_{N k}(E) .
$$

If $E_{0}$ is an interval, then (4.14) is true even if the zeros of $W(z)$ are in E. Furthermore in this case

$$
\phi(T, W): M_{1}^{i}\left(E_{0}\right) \rightarrow M_{N}^{i}(E) \quad(i=0,1) .
$$

PROOF. We shall begin with the case when $E_{0}$ is an interval which we shall take without loss of generality, as $[-1,1]$. We shall also allow that the zeros of $W(z)$ are in $E$. Since $E_{0}=[-1,1], \tilde{a}_{n}^{0}=\frac{1}{2}, \tilde{b}_{n}^{0}=0$, and $\left|T\left(z_{i}\right)\right| \geq 1$, and we have (Chihara [10, Theorems (III.6.4), (IV.2.1), and (IV.2.4)])

$$
\lim _{n \rightarrow \infty} \frac{p_{n}\left(T\left(z_{i}\right) ; \mu_{0}\right)}{p_{n+1}\left(T\left(z_{i}\right) ; \mu_{0}\right)}=\frac{1}{T\left(z_{i}\right)+\sqrt{T\left(z_{i}\right)^{2}-1}}
$$


where the square root is such that $\left|x+\sqrt{x^{2}-1}\right|>1$ when $x \in \mathbf{C} \backslash[-1,1]$. Consequently we have from (4.5), (4.6), and (4.7) that

$$
\lim _{n \rightarrow \infty} a_{(n+1) N}=\tilde{a}_{N}
$$

and

$$
\lim _{n \rightarrow \infty} p_{N-2}^{(n N+1)}(z) \rightarrow q_{N-2}^{(1)}(z)
$$

where the above limit is uniform on compact subsets of C. Here $q_{N-2}^{(1)}$ is some polynomial of degree $N-2$. Since $\hat{p}_{N-1}^{(n N+1)}(z)=\hat{p}_{N-1}^{(1)}(z)=\hat{q}_{N-1}^{(1)}$, we can use (4.10), (4.11), and (4.12) to calculate $\tilde{a}_{i}, i=2,3, \ldots, N-1$, and $\tilde{b}_{i}, i=1,2, \ldots, N-1$. That $\tilde{b}_{0}=\tilde{b}_{n N}$ follows from (3.7) and we find, by equating coefficients of $z^{N-2}$ in (3.10) then taking the limit, that

$$
\tilde{a}_{1}^{2}=\frac{1}{c}\left\{\beta_{2}-\alpha_{2}-\frac{\left(\beta_{1}-\alpha_{1}\right)}{c} \beta_{1}\right\}-\tilde{a}_{N}^{2} .
$$

(Here we have used the fact that the $\left\{\tilde{a}_{n}\right\}$ and $\left\{\tilde{b}_{n}\right\}$ are periodic.) This gives us (4.13) in the case when $E_{0}$ is an interval even if the zeros of $W(z)$ are in $E$. To prove the general case we note that since $z_{i} \notin E,\left|T\left(z_{i}\right)\right|>1$. Let $q_{n}^{0}(x)$ be the orthonormal polynomials associated with $\left\{\tilde{a}_{n}^{0}\right\}$ and $\left\{\tilde{b}_{n}^{0}\right\}$. Then it is easy to show $[\mathbf{1 2},(\mathrm{II} .22)]$ that

$$
\lim _{n \rightarrow \infty} \frac{q_{n k+s}^{0}(x)}{q_{n k+s+1}^{0}(x)}=f_{s}(x), \quad s=0,1,2, \ldots, k-1,
$$

uniformly on compact sets of $\mathbf{C} \backslash[-1,1]$. By the definition of the class $M_{k}\left(E_{0}\right)$ it follows from Poincaré's theorem that

$$
\lim _{n \rightarrow \infty} \frac{p_{n k+s}\left(x ; \mu_{0}\right)}{p_{n k+s+1}\left(x ; \mu_{0}\right)}=f_{s}(x)
$$

uniformly on compact sets of $\mathbf{C} \backslash[-1,1]$. Consequently

$$
\lim _{n \rightarrow \infty} \frac{p_{n k+s}\left(T\left(z_{i}\right) ; \mu_{0}\right)}{p_{n k+s+1}\left(T\left(z_{i}\right) ; \mu_{0}\right)}=f_{s}\left(T\left(z_{i}\right)\right) \text {. }
$$

If we examine the subsequences $\left\{a_{(n k+s+1) N}: n=0,1,2, \ldots\right\}$ and $\left\{p_{N-2}^{((n k+s) N+1)}(x)\right.$ : $n=0,1,2\}(s=0,1,2, \ldots, k-1)$, we can use (4.16) to repeat the same reasoning as before and (4.14) follows.

To show (4.15) we note that from (4.5)-(4.7) and (4.10)-(4.12) it is sufficient to show that

$$
\sum_{n=1}^{\infty} n^{j}\left|\frac{p_{n}\left(T\left(z_{i}\right) ; \mu_{0}\right)}{p_{n+1}\left(T\left(z_{i}\right) ; \mu_{0}\right)}-\frac{1}{T\left(z_{i}\right)+\sqrt{T\left(z_{i}\right)^{2}-1}}\right|<\infty, \quad j=0,1 .
$$

Following [14] we let $\rho(x)=x+\sqrt{x^{2}-1}$ and note that $\rho(x)$ satisfies the following equation:

$$
\rho(x)=2 x-1 / \rho(x) .
$$

From (2.11) we have that for $\mathbf{C} \backslash[-1,1]$

$$
\frac{p_{n}\left(x ; \mu_{0}\right)}{p_{n-1}\left(x ; \mu_{0}\right)}=\frac{z-b_{n-1}}{a_{n}}-\frac{a_{n-1}}{a_{n}} \frac{p_{n-2}\left(x ; \mu_{0}\right)}{p_{n-1}\left(x ; \mu_{0}\right)} \text {. }
$$


Subtracting (4.18) from (4.19) yields

$$
\begin{aligned}
\frac{p_{n}\left(x ; \mu_{0}\right)}{p_{n-1}\left(x ; \mu_{0}\right)}-\rho(x)= & \frac{2 z}{a_{n}}\left(\frac{1}{2}-a_{n}\right)-\frac{b_{n-1}}{a_{n}}+\left(1-\frac{a_{n-1}}{a_{n}}\right) \frac{1}{\rho(x)} \\
& +\frac{a_{n-1}}{a_{n}} \frac{1}{\rho} \frac{p_{n-2}\left(x ; \mu_{0}\right)}{p_{n-1}\left(x ; \mu_{0}\right)}\left(\frac{p_{n-1}\left(x ; \mu_{0}\right)}{p_{n-2}\left(x ; \mu_{0}\right)}-\rho(x)\right) .
\end{aligned}
$$

Since $p_{n-2}\left(x ; \mu_{0}\right) / p_{n-1}\left(x ; \mu_{0}\right) \rightarrow 1 / \rho(x)$ uniformly on compact subsets of $\mathbf{C} \backslash E_{0}$, for any given compact set $K\left(K \cap E_{0}=\varnothing\right)$ and $\varepsilon$ there exists an $N$ such that

$$
\left|\frac{1}{\rho(x)} \frac{p_{n}\left(x ; \mu_{0}\right)}{p_{n-1}\left(x ; \mu_{0}\right)}\right| \leq\left(\frac{1}{|\rho(x)|-\varepsilon}\right)^{2}
$$

for all $n \geq N$ and $x \in K$. Setting

$$
\Delta(n)=\left|\frac{1}{2}-a_{n}\right|+\left|\frac{1}{2}-a_{n-1}\right|+\left|b_{n-1}\right|
$$

and

we find from (4.20) and (4.21) that

$$
\delta=\frac{1}{(|\rho|-\varepsilon)}<1
$$

$$
\left|\frac{p_{n}\left(x ; \mu_{0}\right)}{p_{n-1}\left(x ; \mu_{0}\right)}-\rho(x)\right| \leq \delta^{2}\left|\frac{p_{n-1}\left(x ; \mu_{0}\right)}{p_{n-2}\left(x ; \mu_{0}\right)}-\rho(x)\right|+O(\Delta(n)) .
$$

Repeated application of this inequality gives

$$
\left|\frac{p_{n}\left(x ; \mu_{0}\right)}{p_{n-1}\left(x ; \mu_{0}\right)}-\rho(x)\right|=O\left[\sum_{k=1}^{n} \delta^{(2 n-2 k)} \Delta(k)\right],
$$

where we set $a_{i}=0$ for $i \leq 0$. If we choose $K$ so that $T\left(z_{i}\right) \in K, i=1,2, \ldots, N-1$, the assertion will have been proved if we can show that

$$
I=\sum_{n=1}^{\infty} n^{j} \sum_{k=1}^{n} \delta^{2 k-2 n} \Delta(k)<\infty, \quad j=0,1 .
$$

Interchanging summations in the above equation gives

$$
I=\sum_{k=1}^{\infty} \Delta(k) \delta^{-2 k} \sum_{n=k}^{\infty} n^{j} \delta^{2 n}, \quad j=0,1 .
$$

If we are in the class $M_{1}^{0}$, then

$$
I=\sum_{k=1}^{\infty} \Delta(k) /\left(1-\delta^{2}\right)<\infty
$$

If we are in the class $M_{1}^{1}$, then

$$
I=\frac{1}{\left(1-\delta^{2}\right)^{2}} \sum_{k=1}^{\infty}\left(k-(k-1) \delta^{2}\right) \Delta(k)
$$

which gives the result.

REMARK 5. For other possible rates of convergence of the coefficients see MátéNevai-Totik [19].

By using (2.8), Theorem 7 and Theorem 8 one shows that asymptotic formulas generating functions, etc. for the known polynomial system $p_{n}\left(x ; \mu_{0}\right)$ can be carried over to give corresponding formulas for the system $p_{n}(x ; \mu)$. A simple example of the above is the following theorem. 
THEOREM 10. Suppose that (4.14) holds. Then there exists a constant $A$ such that for $j=0,1,2, \ldots, N-1$

$$
\left\|p_{n N+j-1}^{(1)}(x ; \mu)\right\|_{\infty} \leq A \max \left\{\left\|p_{n}^{(1)}\left(x ; \mu_{0}\right)\right\|_{\infty},\left\|p_{n-1}^{(1)}\left(x ; \mu_{0}\right)\right\|_{\infty}\right\}
$$

and if $W(z) \neq 0$ for $z \in E$ then there exist a constant $B$ such that for $j=$ $0,1,2, \ldots, N-1$

$$
\left\|p_{n N+j}(x ; \mu)\right\|_{\infty} \leq B \max \left\{\left\|p_{n}\left(x ; \mu_{0}\right)\right\|_{\infty},\left\|p_{n+1}\left(x ; \mu_{0}\right)\right\|_{\infty}\right\} .
$$

PROOF. Let

$$
\begin{gathered}
C_{1}=\max _{0 \leq i<N} \max _{k} \sup _{z \in E}\left|p_{i}^{(k)}(z ; \mu)\right|, \\
C_{2}=\max _{n} \frac{a_{n+1}^{0}}{a_{n N+1}}, \quad C_{3}=\max _{0 \leq i<N} \max _{n} \frac{a_{n N+1}}{a_{n N+i+1}} .
\end{gathered}
$$

Then $C_{1}, C_{2}, C_{3}$ are bounded because of the asymptotic periodicity of the recurrence coefficients $\left\{a_{n+1}, b_{n}: n=0,1,2, \ldots\right\}$. From (4.2) one then easily finds

$$
\left\|p_{n N+j-1}^{(1)}(x ; \mu)\right\|_{\infty} \leq \frac{a_{1}}{a_{1}^{0}} C_{2}\left\{C_{3} C_{1}\left\|p_{n-1}^{(1)}\left(x ; \mu_{0}\right)\right\|_{\infty}+C_{1}\left\|p_{n}^{(1)}\left(x ; \mu_{0}\right)\right\|_{\infty}\right\}
$$

from which (4.22) follows. If $W(z) \neq 0$ for $z \in E$, then

$$
C_{4}=\inf _{z \in E}|W(z)|>0
$$

and from (4.1) we find

$$
\left\|p_{n N+j}(x ; \mu)\right\|_{\infty} \leq \frac{C_{2}}{C_{4}}\left\{C_{3} C_{1}\left\|p_{n}\left(x ; \mu_{0}\right)\right\|_{\infty}+C_{1}\left\|p_{n+1}\left(x ; \mu_{0}\right)\right\|_{\infty}\right\},
$$

which leads to (4.23).

V. Potential theory. In this section we will relate some potential-theoretic notions involving the set $E=T^{-1}\left(E_{0}\right)$ to those related to $E_{0}$. If $E$ is a compact set and $\mu$ is a probability measure on $E$, then its (logarithmic) energy is

$$
I(\mu)=\int_{E} \int_{E} \log \frac{1}{|x-y|} d \mu(x) d \mu(y) .
$$

The (logarithmic) potential of $\mu$ is

$$
U(z ; \mu)=\int_{E} \log \frac{1}{|z-x|} d \mu(x) .
$$

If $\Omega_{E}$ is the class of all probability measures on $E$, then the equilibrium energy of $E$ (Robin's constant) is

$$
V(E)=\inf _{\mu \in \Omega_{E}} I(\mu)
$$

and the capacity of $E$ is $C(E)=\exp (-V(E))$. A well-known result in potential theory is that for every compact set $E$ with positive capacity there exists a unique measure $\mu_{E}$ such that $V(E)=I\left(\mu_{E}\right)$. This measure is the equilibrium measure (or Frostman measure) for $E$ (Tsuji [26]). If the capacity of $E$ is positive, then the Green's function $g_{E}(z)$ of $(\mathbf{C} \cup\{\infty\}) \backslash E$ is the only function that is harmonic in $\mathrm{C} \backslash E$, that behaves at $\infty$ as

$$
g_{E}(z)=\log |z|+\text { harmonic function }
$$


and for which

$$
\lim _{\substack{z \notin x \\ z \notin E}} g_{E}(z)=0
$$

for almost every $x$ in $E$. The Green's function is actually given by

$$
g_{E}(z)=-U\left(z ; \mu_{E}\right)-\log C(E)
$$

and

$$
\lim _{z \rightarrow \infty}\left\{g_{E}(z)-\log |z|\right\}=\log \frac{1}{C(E)} .
$$

THEOREM 11. Suppose $C\left(E_{0}\right)>0$ and let $E=T^{-1}\left(E_{0}\right)$. Then

$$
g_{E}(z)=g_{E_{0}}(T(z)) / N
$$

and in particular

$$
C(E)=\left\{C\left(E_{0}\right) / c\right\}^{1 / N} .
$$

If $\mu_{E_{0}}$ is the equilibrium measure on $E_{0}$, then the equilibrium measure $\mu_{E}$ on $E$ can be obtained from $\mu_{E_{0}}$ by taking $W(z)=T^{\prime}(z) / N$.

PROOF. The function $g_{E_{0}}(T(z)) / N$ is harmonic in $\mathbf{C} \backslash E$, in the neighborhood of infinity it behaves as

$$
\begin{aligned}
\frac{1}{N} g_{E_{0}}(T(z)) & =\frac{1}{N}\left\{\log |T(z)|+\log \frac{1}{C\left(E_{0}\right)}\right\}+\text { harmonic function } \\
& =\log |z|+\frac{1}{N} \log c+\frac{1}{N} \log \frac{1}{C\left(E_{0}\right)}+\text { harmonic function }
\end{aligned}
$$

and

$$
\lim _{\substack{z \rightarrow E \\ z \notin E}} \frac{1}{N} g_{E_{0}}(T(z))=\lim _{\substack{z \rightarrow E_{0} \\ z \notin E_{0}}} \frac{1}{N} g_{E_{0}}(z)=0
$$

almost everywhere. Therefore (5.1) and (5.2) follow immediately. Let $W(z)=$ $T^{\prime}(z) / N$ and take $\mu_{0}$ to be the equilibrium measure $\mu_{E_{0}}$ in $E_{0}$; then the corresponding measure $\mu$ in $E$ satisfies

$$
\begin{aligned}
U(z ; \mu) & =\int_{E} \log \frac{1}{|z-x|} d \mu(x) \\
& =\frac{1}{N} \sum_{i=1}^{N} \int_{E_{0}} \log \frac{1}{\left|z-T_{i}^{-1}(x)\right|} d \mu_{0}(x) .
\end{aligned}
$$

Now

$$
\sum_{i=1}^{N} \log \left|z-T_{i}^{-1}(x)\right|=\log \left|\frac{T(z)-x}{c}\right|
$$

so that

$$
U(z ; \mu)=\frac{1}{N} \int_{E_{0}} \log \frac{1}{|T(z)-x|} d \mu_{0}(x)+\frac{1}{N} \log c
$$

and by (5.1) and (5.2) this shows that $\mu$ is the equilibrium measure $\mu_{E}$ on $E$.

If $E_{0}=[-1,1]$, then the equilibrium measure $\mu_{E_{0}}$ is the arcsine measure

$$
d \mu_{E_{0}}(x)=\frac{1}{\pi} \frac{1}{\sqrt{1-x^{2}}} d x, \quad x \in[-1,1],
$$


and the equilibrium measure on $E=T^{-1}([-1,1])$ becomes by Theorem 8

$$
d \mu_{E}(x)=\frac{1}{N \pi} \frac{\left|T^{\prime}(x)\right|}{\sqrt{1-T(x)^{2}}} d x, \quad x \in E .
$$

Theorem 9 shows that the coefficients for this system are in the class $M_{N}^{1}(E)$. However in this case $p_{n}\left(x ; \mu_{0}\right)=T_{n}(x)=\frac{1}{2}\left(\rho(x)^{n}+\rho(x)^{-n}\right)$ are the Chebyshev polynomials of the first kind. Consequently

$$
\frac{T_{n}\left(T\left(z_{i}\right)\right)}{T_{n+1}\left(T\left(z_{i}\right)\right)}= \begin{cases}1 & \text { if } T\left(z_{i}\right)=1, \\ -1 & \text { if } T\left(z_{i}\right)=-1,\end{cases}
$$

and if $\left|T\left(z_{i}\right)\right|>1$ then

$$
\frac{T_{n}\left(T\left(z_{i}\right)\right)}{T_{n+1}\left(T\left(z_{i}\right)\right)}=\frac{1}{\rho\left(T\left(z_{i}\right)\right)}\left[1+O\left(\rho^{-n}\left(T\left(z_{i}\right)\right)\right)\right] .
$$

Thus (4.5)-(4.7) and (4.10)-(4.12) imply the following:

LEMMA 3. Let $p_{n}\left(x ; \mu_{E}\right)$ be the orthonormal polynomials associated with $\mu_{E}$ and let $\left\{a_{n}\right\}$ and $\left\{b_{n}\right\}$ be the coefficients associated with these polynomials. Finally, let $\left\{\tilde{a}_{i}\right\}$ and $\left\{\tilde{b}_{i-1}\right\}(i=1,2, \ldots, N-1)$ be the limits of $a_{n N+i}$ and $b_{n N+i}$ respectively. Then

$$
\left|a_{n N+i}-\tilde{a}_{i}\right|=O\left(\sigma^{-n}\right) \quad \text { and } \quad\left|b_{n N+i}-\tilde{b}_{i}\right|=O\left(\sigma^{-n}\right),
$$

where $\sigma=\min _{z_{i} \notin E_{0}}\left\{\rho\left(T\left(z_{i}\right)\right)\right\}$. Here $z_{i}, i=1,2, \ldots, N-1$, are the zeros of $T^{\prime}(z)$.

REMARK 6. The Szegö class on a set $E$ consists of all measures $\mu(x)$ with absolutely continuous part $\sigma(x)$ having the property that $\ln \sigma(x) \in L_{1}\left(\mu_{E}\right)$. Denote by $S_{E_{0}}$ and $S_{E}$ the Szegö classes on $E_{0}$ and $E$ respectively. Then it follows from (2.6) that

$$
\begin{aligned}
& \frac{1}{N \pi} \int_{E}|\ln \sigma(x)| \frac{\left|T^{\prime}(x)\right|}{\sqrt{1-T(x)^{2}}} d x \\
& \quad=\frac{1}{N \pi} \int_{E}\left\{|\ln | W(x)||+\left|\ln \sigma_{0}(T x)\right|\right\} \frac{\left|T^{\prime}(x)\right|}{\sqrt{1-T(x)^{2}}} d x,
\end{aligned}
$$

which implies that $\phi(T, W): S_{E_{0}} \rightarrow S_{E}$.

REMARK 7 . If we choose $\mu_{0}$ such that it is absolutely continuous on $[-1,1]$ with weight $\sigma_{0}(x)=\frac{1}{\pi}\left(1-x^{2}\right)^{-1 / 2} / \rho(x)$, where $\rho$ is a positive polynomial of degree $l$ on $[-1,1]$, then the $\left\{p_{n}\left(x ; \mu_{0}\right)\right\}$ are Bernstein-Szegö polynomials on $[-1,1]$ (Szegö $[25, \S 2.6])$. The measure $\mu$ on $E=T^{-1}([-1,1])$ is also absolutely continuous on $E$ with weight

$$
\sigma(x)=\frac{1}{\pi} \frac{|W(x)|}{\sqrt{1-T(x)^{2}}} \frac{1}{\rho(T(x))} .
$$

If $W(x)$ has all its zeros at the endpoints of the intervals of $E$, then this measure gives Bernstein-Szegö polynomials on $E$ (Akhiezer [1]). 
VI. Sieved orthogonal polynomials. If we take

$$
T(x)=c \hat{T}_{N}(x)=c 2^{-N+1} T_{N}(x),
$$

where $T_{N}(x)$ is the Chebyshev polynomial of the first kind of degree $N$ and $W=$ $T^{\prime}(x) / N=c \hat{U}_{N-1}(x)=c 2^{-N+1} U_{N-1}(x)$, where $U_{N-1}$ is the Chebyshev polynomial of the second kind of degree $N-1$, then $T$ and $W$ satisfy all the conditions of Lemma 1 if $c \geq 2^{N-1}$. This special case gives rise to so-called sieved orthogonal polynomials, studied by Al-Salam, Allaway, and Askey [2], Charris and Ismail [9] and Ismail [15-17]. The use of the polynomial mapping $T$ gives a new approach to studying sieved orthogonal polynomials and enables one to rediscover many results found by the previously mentioned authors. If we start with a weight function $\sigma_{0}(x)$ on $[-1,1]$, then from $(2.6)$ we see that the orthogonal polynomials on $T^{-1}[-1,1]$ have the weight function

$$
\sigma(x)=c\left|\hat{U}_{N-1}(x)\right| \sigma_{0}\left(c \hat{T}_{N}(x)\right) .
$$

Alternatively letting $\sigma_{0}^{(1)}$ be the weight function for the associated polynomials we find from (2.17) and (2.14) that for appropriate $\sigma_{0}^{(1)}(x)$,

$$
d \mu^{(1)}(x)=\left(\frac{a_{1}^{0}}{a_{1}}\right)^{2} \frac{1}{c\left|\hat{U}_{N-1}(x)\right|} \sigma_{0}^{(1)}\left(c \hat{T}_{N}(x)\right) d x+\sum_{i=1}^{N-1} \rho_{i} \delta\left(x-x_{i}\right) d x
$$

where $x_{i}, i=1,2, \ldots, N-1$, are the zeros of $U_{N-1}(x)$ and

$$
\rho_{i}=\frac{\left(a_{1}^{0}\right)^{2} S\left(c \hat{T}_{N}\left(x_{i}\right) ; \mu_{0}\right)-c \hat{T}_{N}\left(x_{i}\right)+b_{0}^{0}}{a_{1}^{2} c \hat{U}_{N-1}^{1}\left(x_{i}\right)} .
$$

Specific choices of $\sigma_{0}$ or appropriate $\sigma_{0}^{(1)}$ (see Remark 3) (Ultraspherical weights, Pollaczek weights) then give the formula for the weight function obtained in [2, 15-16]. If the spectral measure $\mu_{0}$ on $[-1,1]$ is symmetric, then one can explicitly find the recurrence coefficients for the new orthogonal polynomials in terms of the recurrence coefficients of the polynomials on $[-1,1]$.

THEOREM 12. Suppose $T(x)=c \hat{T}_{N}(x)$ and $W(x)=c \hat{U}_{N-1}(x)$ with $c \geq 2^{N-1}$ and let $b_{n}^{0}=0(n=0,1,2, \ldots)$. Then the recurrence coefficients $\left\{a_{n+1}, b_{n}: n=\right.$ $0,1,2, \ldots\}$ of the new orthogonal system are given by

$$
\begin{gathered}
b_{n}=0, \\
a_{n N+j}=\frac{1}{2}, \quad j=2, \ldots, N-1, \\
a_{n N}^{2}=\frac{a_{n}^{0}}{c} 2^{N-2} \frac{p_{n-1}\left(c 2^{-N+1} ; \mu_{0}\right)}{p_{n}\left(c 2^{-N+1} ; \mu_{0}\right)}, \\
a_{n N+1}^{2}=\frac{1}{2}-\frac{a_{n}^{0}}{c} 2^{N-2} \frac{p_{n-1}\left(c 2^{-N+1} ; \mu_{0}\right)}{p_{n}\left(c 2^{-N+1} ; \mu_{0}\right)} .
\end{gathered}
$$

PROOF. First consider the rational function

$$
\frac{\hat{p}_{N-1}^{(1)}(x ; \mu)}{\hat{p}_{N}(x ; \mu)}=\frac{U_{N-1}(x)}{T_{N}(x)}
$$


where the equality follows from Theorems 1 and 2. The coefficients of the (Jacobi) continued fraction are $\left\{b_{0} ; a_{n}^{2}, b_{n}: n=1, \ldots, N-1\right\}$ and because we are dealing with Chebyshev polynomials we have

$$
\begin{array}{cl}
a_{1}=1 / \sqrt{2} ; & b_{0}=b_{1}=0, \\
a_{j}=\frac{1}{2} ; \quad b_{j}=0, & j=2,3, \ldots, N-1 .
\end{array}
$$

Suppose next that we have proved

$$
a_{n N+j}=\frac{1}{2}, \quad b_{n N+j}=0 \quad(j=2,3, \ldots, N-1) ; \quad b_{n N+1}=0
$$

for every $n=0,1, \ldots, k-1$. Formula (3.10) becomes for $n=k$

$$
a_{k N}^{2} \hat{p}_{N-2}^{(k N+1)}(z ; \mu)+a_{k N+1}^{2} \hat{p}_{N-2}^{(k N+2)}(z ; \mu)=2^{-N+1} U_{N-2}(z) .
$$

Comparing the leading coefficients gives

$$
a_{k N}^{2}+a_{k N+1}^{2}=\frac{1}{2}
$$

and by hypothesis $\hat{p}_{N-2}^{(l N-N+1)}(z ; \mu)=2^{-N+2} U_{N-2}(x)$; therefore

$$
a_{k N+1}^{2} \hat{p}_{N-2}^{(k N+2)}(z ; \mu)=2^{-N+2} U_{N-2}(x)\left\{\frac{1}{2}-a_{k N}^{2}\right\}
$$

from which we find $\hat{p}_{N-2}^{(k N+2)}(z ; \mu)=2^{-N+2} U_{N-2}(x)$. The coefficients in the (Jacobi) continued fraction for

$$
\frac{\hat{p}_{N-2}^{(k N+2)}(x ; \mu)}{\hat{p}_{N-1}^{(k N+1)}(x ; \mu)}=\frac{1}{2} \frac{U_{N-2}(x)}{U_{N-1}(x)}
$$

are $\left\{b_{k N+1} ; a_{k N+j}^{2}, b_{k N+j}: j=2, \ldots, N-1\right\}$ and since we are dealing with Chebyshev polynomials of the second kind we have

$$
\begin{aligned}
& b_{k N+j}=0, \quad j=1, \ldots, N-1, \\
& a_{k N+j}=\frac{1}{2}, \quad j=2, \ldots, N-1 .
\end{aligned}
$$

Also $b_{k N}=0$ by (3.7), so that (6.1) and (6.2) are true for every $n$. Because of (6.1) and (6.2) we have $\hat{p}_{N-2}^{(n N+1)}(z ; \mu)=2^{-N+2} U_{N-2}(z)$ so that (4.7) becomes

$$
a_{n N}^{2}=-\frac{a_{n}^{0}}{c} \frac{2^{N-2}}{U_{N-2}\left(z_{i}\right)} \frac{p_{n-1}\left(T\left(z_{i}\right) ; \mu_{0}\right)}{p_{n}\left(T\left(z_{i}\right) ; \mu_{0}\right)}
$$

where $z_{i}$ is a zero of $U_{N-1}$. Now $U_{N-2}\left(z_{i}\right)=-T_{N}\left(z_{i}\right)= \pm 1$ from which (6.3) follows. The result in (6.4) is a combination of (6.3) and (6.5).

The formulas for the intermediate polynomials $p_{n N+j}(x ; \mu)$ and $p_{n N+j-1}^{(1)}(x ; \mu)$ in Theorem 7 become quite simple. Indeed, because of $(6.1)$ and (6.2) we have for $j=1, \ldots, N-2$

$$
2 a_{(n+1) N} p_{N-j-1}^{(n N+j+1)}(x ; \mu)=U_{N-j-1}(x), \quad p_{j-1}^{(n N+1)}(x ; \mu)=U_{j-1}(x)
$$

so that under the conditions of Theorem 12 we have for $1 \leq j<N$

$$
\begin{aligned}
p_{n N+j}(x ; \mu)=\frac{2^{N-1}}{c} \frac{a_{n+1}^{0}}{a_{n N+1}}\left\{\frac{a_{n N+1}}{a_{n N+n}} U_{N-j-1}(x) p_{n}\left(T(x) ; \mu_{0}\right)\right. \\
\left.+U_{j-1}(x) p_{n+1}\left(T(x) ; \mu_{0}\right)\right\} / U_{N-1}(x)
\end{aligned}
$$


and

$$
\begin{array}{r}
p_{n N+j-1}^{(1)}(x ; \mu)=\frac{1}{\sqrt{2} a_{1}^{0}} \frac{a_{n+1}^{0}}{a_{n N+1}}\left\{\frac{a_{n N+1}}{a_{n N+n}} U_{N-j-1}(x) p_{n-1}^{(1)}\left(T(x) ; \mu_{0}\right)\right. \\
\left.+U_{j-1}(x) p_{n}^{(1)}\left(T(x) ; \mu_{0}\right)\right\} .
\end{array}
$$

The special case $c=2^{N-1}$ gives a mapping such that $T^{-1}([-1,1])=[-1,1]$. The weight function $\sigma$ may vanish at the zeros of the Chebyshev polynomial $U_{N-1}$. As for the associated polynomials we see that for appropriate weight functions $\sigma_{0}^{(1)}$ we may find mass points of $\mu^{(1)}$ in $[-1,1]$. For example if one chooses

$$
\sigma_{0}^{(1)}=\frac{\Gamma\left(\alpha+\frac{3}{2}\right)\left(1-x^{2}\right)^{\alpha}}{\sqrt{\pi} \Gamma(\alpha+1)}, \quad \alpha>0,
$$

then

$$
S\left( \pm 1, \mu_{0}^{(1)}\right)= \pm \frac{\left(\alpha+\frac{1}{2}\right)}{\alpha}
$$

$[\mathbf{2 5}$, p. 77] and we find that

$$
\begin{aligned}
d \mu^{(1)}(x)= & \frac{2\left(a_{1}^{0}\right) \Gamma\left(\alpha+\frac{3}{2}\right)}{\sqrt{\pi} \Gamma(\alpha+1)} \frac{\left(1-T_{N}^{2}(x)\right)^{\alpha}}{\left|U_{N-1}(x)\right|} d x \\
& +\sum_{i=1}^{N-1} \frac{2}{\alpha}\left(\alpha-(\alpha+2)\left(a_{1}^{0}\right)^{2}\right) \sin ^{2}\left(\frac{i \pi}{N}\right) \delta\left(x-\cos \frac{i \pi}{N}\right) d x,
\end{aligned}
$$

where one must choose $\left(a_{1}^{0}\right)^{2} \leq \alpha /\left(\alpha+\frac{1}{2}\right)$ (see Remark 3).

We note that the above calculations easily carry over to the case when $\mu_{0}^{(1)}$ is a Jacobi weight with $\alpha, \beta>0$.

\section{REFERENCES}

1. N. I. Akhiezer, Orthogonal polynomials on several intervals, Dokl. Akad. Nauk SSSR 134 (1960), 9-12; English transl., Soviet Math. Dokl. 1 (1961), 989-992.

2. W. Al-Salam, W. Allaway and R. Askey, Sieved ultraspherical polynomials, Trans. Amer. Math. Soc. 284 (1984), 39-55.

3. A. I. Aptekarev, Asymptotic properties of polynomials orthogonal on a system of contours, and periodic motions of Toda lattices, Mat. Sb. 125 (167) (1984), 231-258; English transl., Math. USSR Sb. 53 (1986), 233-260.

4. M. F. Barnsley, J. S. Geronimo and A. N. Harrington, Almost periodic Jacobi matrices associated with Julia sets for polynomials, Comm. Math. Phys. 99 (1985), 303-317.

5. P. Barrucand and D. Dickinson, On cubic transformations of orthogonal polynomials, Proc. Amer. Math. Soc. 17 (1966), 810-814.

6. J. Bellissard, Stability and instability in quantum mechanics, Trends in the Eighties (Ph. Blanchard, ed.), Singapore, 1985.

7. D. Bessis, J. S. Geronimo and P. Moussa, Function weighted measures and orthogonal polynomials on Julia sets, Constr. Approx. (to appear).

8. D. Bessis and P. Moussa, Orthogonality properties of iterated polynomial mappings, Comm. Math. Phys. 88 (1983), 503-529.

9. J. Charris and M. E. H. Ismail, On sieved orthogonal polynomials, II: random walk polynomials, Canad. J. Math. 38 (1986), 397-415.

10. T. S. Chihara, An introduction to orthogonal polynomials, Gordon and Breach, New York, 1978. 
11. C. de Boor and J. R. Rice, Extremal polynomials with application to Richardson iteration for indefinite linear systems, SIAM J. Sci. Statist. Comput. 3 (1982), 47-57.

12. J. S. Geronimo and W. Van Assche, Orthogonal polynomials with asymptotically periodic recurrence coefficients, J. Approx. Theory 46 (1986), 251-283.

13. Ya. L. Geronimus, On the character of the solutions of the moment problem in the case of a limit-periodic associated fraction, Izv. Akad. Nauk SSSR 5 (1941), 203-210. (Russian)

14. __ On some finite difference equations and corresponding systems of orthogonal polynomials, Zap. Mat. Otdel. Fiz.-Mat. Fak. i Kharkov Mak. Obsc. (4) 25 (1957), 87-100. (Russian)

15. M. E. H. Ismail, On sieved orthogonal polynomials, I: symmetric Pollaczek analogues, SIAM J. Math. Anal. 16 (1985), 1093-1113.

16. __ On sieved orthogonal polynomials, III: orthogonality on several intervals, Trans. Amer. Math. Soc. 294 (1986), 89-111.

17. _ On sieved orthogonal polynomials, IV: generating functions, J. Approx. Theory 46 (1986), 284-296.

18. V. I. Lebedev, Iterative methods for solving operator equations with a spectrum contained in several intervals, Zh. Vychisl. Mat. i Mat. Fiz. 9 (1969), 1247-1252; English transl., USSR Comput. Math. and Math. Phys. 9 (1969), 17-24.

19. A. Máté, P. Nevai and V. Totik, Asymptotics for orthogonal polynomials defined by a recurrence relation, Constr. Approx. 1 (1985), 231-248.

20. P. Moussa, Itérations des polynômes et propriétés d'orthogonalité, Ann. Inst. H. Poincaré Phys. Theor. 44 (1986), 315-325.

21. P. G. Nevai, Orthogonal polynomials, Mem. Amer. Math. Soc. No. 213 (1979).

22. D. G. Pettifor and D. L. Weaire (eds.), The recursion method and its applications, Springer Series in Solid State Sciences 58, Springer-Verlag, Berlin, 1985.

23. Y. Saad, Iterative solution of indefinite symmetric linear systems by methods using orthogonal polynomials over two disjoint intervals, SIAM J. Numer. Anal. 20 (1983), 784-811.

24. M. H. Stone, Linear transformations in Hilbert space, Amer. Math. Soc. Colloq. Publ., vol. 15, Amer. Math. Soc., Providence, R.I., 1928.

25. G. Szegö, Orthogonal polynomials, 4th ed., Amer. Math. Soc. Colloq. Publ., vol. 23, Amer. Math. Soc., Providence, R.I., 1975.

26. M. Tsuji, Potential theory in modern function theory, Chelsea, New York, 1959.

27. J. C. Wheeler, Modified moments and continued fraction coefficients for the diatomic linear chain, J. Chem. Phys. 80 (1984), 472-476.

School of Mathematics, Georgia institute of Technology, Atlanta, GeorGIA 30332 